\title{
Broadband Marine Seismic, Does Acquisition Make a Difference?
}

C. Koeninger* (Schlumberger PTS), M. Bayly (Schlumberger PTS) \& S.L. Ng (Schlumberger PTS)

\section{Introduction}

Expanding the bandwidth of surface seismic data, particularly towards low frequencies, is essential for many exploration and production objectives. Broader band signals, both in land and marine environments have marked benefits for imaging deeper targets, imaging through absorptive overburdens, and especially inversion for rock properties. Various methods have been proposed and implemented to expand seismic bandwidth; these include both acquisition and signal processing methods. A question that is often asked is how much difference does changing the acquisition geometry make? In this paper, we present a case study of a consistent, experimental offshore dataset in Southeast Asia. This data consists of a single boat pass of different cable depth configurations. These data were then processed with their appropriate deghosting methods and results compared. In addition, we examine methods for evaluating the success of these methods and their potential pitfalls. The key determinant of the eventual bandwidth of surface seismic data is the convolution of the source, near surface effects (free surface ghosts in the marine situation), the overall earth attenuation, and the level of additive environmental noise. Some of these effects can be modified by changes in the field acquisition geometry or at least, deterministically compensated for. The noise level may constrain or limit the capacity of signal processing tools to compensate for the "field" effects. When evaluating the raw and processed data it is wise to use various types of analysis and displays. Simple seismic amplitude stack sections and associated spectra can be misleading. Spectral "split" plots and inversion of the seismic data is often more indicative of success.

In marine seismic acquisition, the free-surface ghost effect is one factor that can strongly impact the data bandwidth characteristics according to streamer and source depth. It is also a factor that could easily be adjusted in the survey design. Shallow towing favors the higher frequency response at the expense of low frequencies, whilst deeper towing favors the lower frequencies, at the expense of higher frequencies. Moreover, a deeper tow typically has lower levels of swell noise. At very low frequencies, the critical two octaves between two to eight Hertz, the level of ambient towing noise rises, the "DC notch" strengthens, and the power output of airgun sources declines. This combination provides both the biggest challenge and the opportunity of bandwidth expansion.

\section{Marine Broadband Acquisition Techniques}

A solution to this "barrier" is to create "notch diversity" and preferably reduce environmental noise. By deepening the cable the frequency where noise overcomes signal can be shifted lower than that of a typical $6-8 \mathrm{~m}$ tow depth. The combination of designing field methods providing notch diversity, coupled with careful data processing, can improve bandwidth particularly at very low frequencies. We shall illustrate these methods with a comparative field test recently conducted in Southeast Asia. These examples are also processed with suitable corrections and compensation. From this test we can draw some conclusions as to applicability of these notch diversity methods relative to recent practice and survey needs.

\section{Field Test}

In the experimental data, multiple cables were towed by the vessel at differing depths. Inducing notch diversity by slanting the cable (ref. Ray and Moore, 1982) is one of the tests. However this presents a challenge to data processing, the amplitude and phase response of the recorded data will progressively change over offset. Unless compensated for, this will bias multichannel processes that work across the entire spread. Of particular concern would be velocity analysis, SRME based demultiple, and prestack AVO analysis. In this experiment, the slanted cable data is processed using a proprietary single streamer deghosting (SSD) technique at an early stage of processing immediately after noise attenuation. The overunder cable configuration (ref. Haggerty, 1956) is another test, this data is de-ghosted using a proprietary, optimal deghosting technique (ODG) (ref. Ozdemir et. al., 2003). The 16 metre deep cable is also processed with SSD and residual spectral shaping for the remaining but weak notch at around $48 \mathrm{~Hz}$. 


\section{Results and Analysis}

In this experiment, all broad band methods tested increased the band width compared to the "traditional" approach. An additional octave or more of signal is added at the low frequency end of the spectra. Varying amounts of high frequency enhancement have been achieved. The over-under technique provides approximately $30 \mathrm{~Hz}$ more than the traditional shallow "flat tow" method while the slant cable method indicated a $15 \mathrm{~Hz}$ increase.

Overall the dual cable method provides the broadest bandwidth consistent with it having two measurements along the entire offset range. The slanted cable method provides the second best result. The deep cable result is surprisingly good and it was noticed that the high frequency notch is not as severe as may be expected. This is most likely due to the fact that even with a nominal constant cable depth there is some notch diversity in the data as the sea level rises and falls. This data was collected in quite rough conditions when the average swell range was $\pm 1.5 \mathrm{~m}$, hence the ghost can be considered "fuzzy" especially when averaged via CMP stacking and prestack migration.

However, caution should be exercised as further spectral shaping techniques can be applied that reduce the differences that the various field acquisition techniques "ghost filter" imposes. As long as the signal to noise level in a dataset is good, the visual impact (the top $20 \mathrm{~dB}$ of the spectra) of the imposed free surface ghost filter can be reduced.

The expanded bandwidth and especially the ultra low frequencies are very useful not only for penetration through deep or highly absorptive media but also for inversion of various forms. They significantly reduce the dependency upon low frequency modeling in the case of inversion for acoustic impedance and are essential for emerging technologies such as full waveform inversion (FWI).

\section{Conclusions}

Comprehension of the principles governing the bandwidth of both signal and noise of seismic data enables the design of field systems to provide optimal data for modern processing techniques. The broadband acquisition methods tested here do make a difference to the acquired data. However a complimentary processing technique is also necessary to complete the challenge. In a perfect noise free world this would not be the case as spectral shaping could overcome any free surface ghost amplitude attenuation and the phase effects can also be modeled and reversed. However, environmental noise is always present to some degree and noise mitigation via deeper tows and notch diversity via field design can assist in improving data. Critical evaluation of the results demonstrates that broadband seismic can play a significant role in addressing the needs of exploration and development surveys in Asia and beyond. The exact method employed is most likely to be chosen based on operational and project objectives.

\section{Acknowledgements}

The authors thank WesternGeco management for financing the acquisition and processing of the experiment and permission to publish this paper. The authors also thank PETRONAS for providing the test location.

\section{References}

Haggerty, P.E. [1956] Method and apparatus for cancelling reverberations in water layers. US patent No $2,757,356$.

Ray, C.H. and Moore, N.A. [1982] High resolution, marine seismic stratigraphic system. US patent No. $4,353,121$.

Ozdemir, A.K., Caprioli, P., Ozbek, A., Kragh, E. and Robertson, J.O.A. [2008] Optimized deghosting of over/under towed-streamer data in the presence of noise. The Leading Edge, 27, 190-199. 